\title{
Development of the Rabbit (Oryctolagus cuniculus) Metanephros
}

\section{W. Gaber}

Department of Anatomy, Histology and Embryology, Faculty of Veterinary Medicine, Assiut University, Assiut, Egypt.

\section{Abstract}

Thirty-seven rabbit embryos at embryonic days $(E)$ 10-30 were used to study the morphogenesis of the metanephros by light microscope. The primordium of the metanephros appears at E 12 within the pelvic cavity as a spherical ureteric bud capped by metanephrogenic tissue. The nephrogenic spherules and the renal vesicles develop at $\mathrm{E} 15$ while formation of the renal tubules and differentiation of the glomerular capsule take place at E 16. At E 18, differentiation of the glomerular capillaries occurs and the proximal and distal convoluted tubules are clearly first time demarcated. Positive alkaline phosphatase activity and PAS-positive material are confined to the metanephric corpuscles and the luminal border of the proximal convoluted tubules. In addition, the collecting tubules show large masses of intracytoplasmic PASpositive granules. The rabbit metanephros is smooth unipapillary with indistinct renal columns and pyra- mids. It undergoes migration until reaches its permanent position at $\mathrm{E}$ 30 where the right metanephros is located opposite to the level of the $2^{\text {nd }}-4^{\text {th }}$ lumbar vertebrae and the left one is situated opposite to the level of the $3^{\text {rd }}-5^{\text {th }}$ lumbar vertebrae. This migration is accompanied by rotation for about 135 degrees resulting in a dorsomedially located hilus. In conclusion, the stages of development of the rabbit metanephros are similar to those in other mammals but they maintain a delayed sequence.

Keywords: Rabbit, Metanephros, Development, Embryos.

\section{Introduction}

Metanephros is the primordium of the permanent kidney. It develops from two sources; ureteric bud (metanephric diverticulum) which forms the collecting part and metanephrogenic mass of intermediate mesoderm which gives the secretory part i.e. nephron (Huber, 1905; 
Hamilton and Mossman, 1976; Kaufman, 1992; Imam, 2005). Many aspects of the development of the metanephros have been investigated by histologists and embryologists in different species; (Aoki, 1966; Rao and Padmini, 2012) in human, Canfield (1980) in bovine, Imam (2005) in camel and (Abbas and Rabie, 2012; Fayez et al., 2014) in rabbit.

The rabbit is the only known mammal in which the tubules can be separated from kidney slices with the basement membrane intact, a factor that has justified its use in many studies involving renal tubule physiology. Information about the known differences between the kidney of the rabbit and the kidneys of other mammals is therefore of special importance (Brewer, 2006). This article represents a light microscopic study on the development of the metanephros in rabbits during the prenatal life and aims to focus on its origin, early development and migration, in addition to the histological and histochemical changes of the nephron and the duct system during different embryonic stages.

\section{Materials and Methods}

Thirty seven normal New Zealand white rabbit embryos at embryonic days $(E)$ 10-30 were collected from the Research Farm of Faculty of Agriculture, Assiut University, Egypt

J. Vet. Anat.
(Table 1). Ethical approval was obtained from Animal Care and Use Committee of Assiut University. The pregnant rabbits were sacrificed at various periods of gestation and the embryos were removed shortly after evisceration. The embryos were fixed in 10\% neutral buffered formalin or Bouin's fluid. For histological and histochemical studies, the embryos aged 10-26 days were taken as a whole while in those aged 28 and 30 days, only the metanephri were used. The fixed specimens were dehydrated in graded alcohol series, cleared, embedded in paraffin and serially sectioned at 3-5 $\mu \mathrm{m}$ thick. The obtained sections were stained with the following stains: Harris haematoxylin and eosin stain (Harris, 1900) for general histological studies, Periodic Acid Schiff reaction (PAS) (McManus, 1946) for detection of glycoproteins and Gomori calcium method (Gomori, 1952) for detection of alkaline phosphatase activity. The prepared sections were demonstrated by light microscope. For studying the position of the metanephri at full term, 3 embryos at $E 30$ were dissected.

\section{Results}

At E 10 and E 11, the ureteric bud could not be observed at the pelvic part of the mesonephric duct.

At $E$ 12, the primordium of the metanephros could be observed as a

Vol. 10, No. 2, (2017) 109 - 130 
spherical ureteric bud capped by metanephrogenic tissue. The ureteric bud arises from the dorsal aspect of the caudal part of the mesonephric duct before its entrance into the urinary bladder. The metanephros primordium is located within the pelvic cavity, caudal to the mesonephros for some distance and is related dorsally to the aorta, ventrally to the rectum and ventrolaterally to the umbilical artery. The metanephrogenic tissue consists of an overcrowded inner layer and a loose outer layer of mesenchyme. This mesenchyme consists of small branched cells with little amount of cytoplasm and large oval nuclei (Fig. 1).

At $E$ 13, the primordium of the metanephros extends more cranially to be located just caudal to the mesonephros. The metanephros becomes enlarged and elongated craniocaudally. The first division of the ureteric bud could be observed consisting of cranial and caudal branches surrounded by the metanephrogenic tissue. These branches are lined by simple columnar epithelium with rounded or oval basely located nucleus and acidophilic cytoplasm (Fig. 2). It becomes stratified in some areas and many mitotic divisions could be noticed.

At $E$ 14, the metanephros is compressed between the last lumbar somites dorsally and the caudal end of the mesonephros ventrally (Fig. 3 ). It is bean shaped where it has dorsal and ventral surfaces, cranial and caudal extremities, convex lateral border and slightly concave medial border. The ureter leaves the middle of the medial border. The two metanephri are opposite to each other craniocaudally and dorsoventrally. They are related medially to the aorta and projected laterally into the celomic cavity (Fig. 4). The ureteric bud gives off the second division. Each branch of the ureteric bud is capped by its own metanephrogenic tissue and all are surrounded by loose mesenchyme containing some red blood cells. The metanephros is surrounded externally by a thin layer of mesenchyme, which represents the prospective renal capsule (Fig. 5). The branches of the ureteric bud showed positive PAS reaction while the metanephrogenic tissue was PAS negative (Fig. 6).

At E 15, the metanephros is located dorsal to the mesonephros, just caudal to its middle (Fig. 7). The right metanephros is situated somewhat cranial and dorsal to the level of the left one (Fig. 8). The metanephros differentiates into an outer dense cortex and an inner loose medulla. The medulla contains few collecting tubules surrounded by large amount of intertubular mesenchyme. Each group of 
the collecting tubules is capped by its own metanephrogenic tissue forming together the primitive renal lobes, which are separated from each other by interlobar mesenchyme containing many red blood cells. Outgrowths of the collecting tubules extend within the cortex with their terminal branches breaking down the metanephrogenic tissue into smaller separate portions. The cells of the metanephrogenic tissue around the collecting tubules are arranged in several layers. They are closely packed with large deeply stained nuclei. At the angle between the collecting tubule and its terminal branches, there are small nephrogenic spherules. Some of these spherules develop a narrow lumen in its center forming renal vesicles (Fig. 9). The collecting tubules showed PAS-positive material while the metanephrogenic tissue, the nephrogenic spherules and the renal vesicles were PAS negative.

At $E$ 16, the metanephros extends a bit cranially to be located dorsal to the middle of the mesonephros. The nephrogenic tissue of the adjacent primitive renal lobes becomes completely fused forming a continuous outer nephrogenic layer giving the metanephros a smooth outer surface. Within the cortex, most of the metanephrogenic spherules develop into renal vesicles while; others remain solid and somewhat peripher- ally located. Some of the renal vesicles become elongated into renal tubules and acquire different shapes; comma-shape and Sshape. Few of the ends of these tubules, which are close to the terminal branches of the collecting tubules, are continuous with them (Fig. 10). The other ends become ampullated and invaginated by mesenchymal mass to form a crescent-shaped glomerular capsule. This mesenchymal mass contains some red blood cells. The glomerular capsule has an outer parietal and an inner visceral layer with glomerular cavity inbetween. This cavity shows different width. The epithelium of the parietal layer is simple squamous while that of the visceral layer is low columnar (Fig. 11).

The renal tubules have narrow lumen and are lined by simple columnar epithelium with oval basely located nuclei and deeply stained cytoplasm. The terminal branches of the collecting tubules within the cortex are small, have wide lumen and lined by simple low columnar epithelium with oval basely located nuclei and pale cytoplasm. The intertubular mesenchyme contains some red blood cells. The part of the renal tubule nearby the glomerular capsule is the prospective proximal convoluted tubule. The other part near the terminal branches of the 
collecting tubule is the prospective distal convoluted tubule and the middle part will form the loop of Henle.

The medulla contains stems of the collecting tubules surrounded by large amount of loose mesenchyme. These collecting tubules are large, have wide lumen and lined by high columnar epithelium with oval centrally located nuclei and pale cytoplasm. The cells of the collecting tubules showed masses of PASpositive intracytoplasmic granules while the metanephrogenic tissue, the nephrogenic spherules, the renal vesicles as well as the renal tubules showed negative PAS reaction.

At E18, the cranial two thirds of the metanephros become cranial to the level of the mesonephros therefore; it is related ventrally to the liver on the right side and the stomach on the left (Figs. 12\&13). The hilus becomes located somewhat dorsomedially (Fig. 14).

The metanephros is covered externally by connective tissue capsule. The outer layer of the cortex is represented by a thin layer of metanephrogenic tissue followed by the renal tubules. The peripheral renal tubules remain undifferentiated while the inner ones, towards the medulla, become differentiated into proximal convoluted tubules, loop of
J. Vet. Anat.
Henle and distal convoluted tubules. The undifferentiated renal tubules have narrow lumen and are lined by simple columnar epithelium with large oval vesicular nuclei and dark cytoplasm (Fig. 15). The proximal convoluted tubules are numerous, large and lined by large cuboidal epithelium with rounded vesicular nuclei and eosinophilic cytoplasm with uneven luminal border. The distal convoluted tubules are few, smaller, have wide lumen and lined by cuboidal epithelium with dark cytoplasm.

Near the medulla, the mesenchymal mass, which invaginates into the glomerular capsule is differentiated into glomerular capillaries, some contain red blood cells. The visceral layer of glomerular capsule becomes lined by podocytes, which come in contact with the endothelium of the glomerular capillaries. The glomerulus and the glomerular capsule forming the metanephric corpuscles (Fig. 16).

The terminal branches of the collecting tubules between the undifferentiated renal tubules are lined by simple low columnar epithelium with oval basely located nuclei and pale cytoplasm while, that deeper in the cortex are lined by simple columnar epithelium with rounded centrally located nuclei and pale cytoplasm. The renal pelvis is lined by simple columnar epithelium with 113 Vol. 10, No. 2, (2017) $109-130$ 
rounded apically located nuclei and pale cytoplasm. In some areas, the epithelium was stratified (Fig. 17). Masses of PAS-positive granules could be seen within the cytoplasm of the collecting tubules. There was a slight PAS-positive material in the luminal border of some proximal convoluted tubules while the metanephric corpuscles, the distal convoluted tubules, the loop of Henle and the undifferentiated renal tubules showed negative PAS reaction (Fig. 18). Slight alkaline phosphatase activity was confined to the luminal border of the proximal convoluted tubules, the visceral epithelium of the glomerular capsule and the endothelium of the glomeruli (Fig. 19).

At E 20, the metanephros is related caudally to the degenerated mesonephros, cranially to the liver on the right side and the stomach on the left. Ventrally, it is related to the liver on the right side and the intestine on the left. The hilus is located dorsomedially. The layer of the metanephrogenic tissue in the outer part of the cortex becomes thinner and interrupted by renal tubules. The proximal and distal convoluted tubules increase in density on the expense of the outer undifferentiated renal tubules.

The renal pelvis gives off papillary ducts which branch into stems of the collecting tubules (Fig. 20). In the mesenchyme close to the renal pelvis, some small renal tubules lined by cuboidal epithelium could be observed. Transitional epithelium could be detected in some areas of the lining epithelium of the renal pelvis. It consists of; basal layer of high cuboidal cells with rounded apically located nuclei, middle layer consists of several layers of polyhedral cells, the most outer one consists of pear-shaped cells, and a superficial layer of large cuboidal cells with dome-shaped apical surface (Fig. 21). The collecting tubules showed large masses of PASpositive granules in their cytoplasm. Moderate PAS reaction and alkaline phosphatase activity could be observed within the luminal border of all proximal convoluted tubules as well as the juxtamedullary metanephric corpuscles.

At E 23 and E 24, the demarcation between the cortex and the medulla is well distinct (Fig. 22). The layer of the metanephrogenic tissue could not be observed. The medullary rays could be detected extending in the cortex towards the renal capsule forming the radiated part of the cortex, which alternates with the convoluted part of the cortex (Fig. 23). The interlobar blood vessels and the renal columns are indistinct consequently; the renal pyramids could not be detected. Within the medulla, 
the arteria recti could be observed extending between the collecting tubules towards the renal pelvis (Fig. 24). The relative position of the main structures in the hilus is that the renal artery is located craniodorsally and the ureter is caudoventrally while the renal vein is inbetween (Figs. 25\&26).

At E 26 and $E$ 28, the metanephros reaches the adult structure. The radiated and convoluted parts of the cortex become well distinct (Fig. 27). The interlobar blood vessels could be clearly observed while the renal columns are still indistinct. The stems of the collecting tubules are directed towards the renal pelvis to open into the papillary ducts, which open on a common renal papilla that is projected within the large renal pelvis (Fig. 28). The stems of the collecting tubules and the papillary ducts are lined by tall columnar epithelium with centrally located nucleus (Fig. 29). The epithelium of the common renal papilla and the renal pelvis is transitional in type (Fig. 30).

At E 24 and E 26, strongly positive alkaline phosphatase activity and much PAS-positive material were confined to the metanephric corpuscles and the luminal border of the proximal convoluted tubules (Figs. 31\&32). The collecting tubules showed very large masses of intra- cytoplasmic PAS-positive granules (Fig. 33).

At $E$ 30, the right metanephros is located opposite to the level of the $2^{\text {nd }}-4^{\text {th }}$ lumbar vertebrae within the renal impression of the liver, which is very deep and formed by the caudate process only. The left metanephros is situated opposite to the level of the $3^{\text {rd }}-5^{\text {th }}$ lumbar vertebrae (Fig. 34).

\section{Discussion}

The stages of development of the metanephros in rabbit are similar to those in other mammals but they maintain a delayed sequence where, according to the present study, the primordium of the metanephros is firstly observed at E 12 (about the end of the first half of gestation) consisting of ureteric bud surrounded by metanephrogenic tissue. The ureteric bud originates from the mesonephric duct before its entrance in the urinary bladder while, in human according to Sadler (2000), the primitive metanephros appears so early at the fifth week of gestation as an outgrowth from the mesonephric duct before its entrance in the cloaca. Due to late development of the metanephros in rabbit, the cloaca has been differentiated into rectum, urinary bladder and urogenital sinus therefore, at this time; in rabbit the mesonephric duct opens in the urinary bladder 
but not in the cloaca as in human. At early stage of development ( $E$ 12), the metanephros is spherical in shape and at E 13, it is elongated therefore, it has cranial and caudal ends. At E 14, the metanephros acquires its adult bean shape where, at this age it is compressed between the lumbar somites and the mesonephros so it has dorsal and ventral surfaces. In addition, due to the differential growth of its borders, the medial border becomes slightly concave and the lateral border becomes convex.

Nickel et al. (1973) stated that, during development of the metanephros, the type of the kidney depends upon the degree of fusion of the cortex and medulla of the adjacent lobes. If the fusion of the cortex is incomplete, the kidney becomes fissured as in ox but if the fusion is complete, the kidney becomes smooth as in horse, dog and small ruminants. On the other hand, if the papillae of the adjacent lobes are partially fused, the kidney is multipapillary as in ox and pig but in that of the horse, dog and small ruminants, the papillae are completely fused therefore; the kidney is unipapillary. In this respect, the results of the current work reveal that the kidney of the rabbit is smooth unipapillary as horse, dog and small ruminants where at $E 15$, the nephrogenic tissue of the adjacent primitive lobes of the kidney is separated by interlobar mesenchyme but at $E$ 16, this nephrogenic tissue completely fuses forming an outer smooth surface. On the other hand, at $E 26$, the papillae are completely fused forming a common renal papilla i.e. unipapillary kidney. These results are in accordance with Abbas and Rabie (2012) in rabbit fetuses.

The kidney undergoes a remarkable change in position during its development. It migrates from the pelvic to the lumbar region (Gruenwald, 1943; McGeady et al., 2006) and this is considered an exception to the general rule in developmental topography, since it migrates upword along the vertebral column, instead of downword like the other viscera (Jackson, 1909; Arey, 1965). Many authors have studied the migration of the kidney; Leeson and Baxter (1957) in rabbit, Canfield (1980) in bovine and Imam (2005) in camel. There is a great controversy about the mechanism of displacement of the kidney. Some authors assume that it is an active growth as the anlage elongates, extending forward along the line of least resistance in the loose mesenchyme of the space bounded by the aorta dorsally, the rectum ventrally, and the umbilical arteries laterally (Jackson, 1909), others believe that this displacement is probably due to a 
diminution of the body curvature (Gruenwald, 1943), while others stated that in addition to being an active cranial migration it is also due to differential growth of the skeletal and muscular structures in the lumbo-sacral region (Patten, 1953; Arey, 1965; McGeady et al., 2006).

Concerning migration, according to the current study, the metanephros of rabbit embryos is located in the pelvic cavity at E12 but at E 14, it is located dorsal to the caudal end of the mesonephros i.e. in the caudal part of the lumbar region then at $\mathrm{E}$ 15 it extends a bit cranially to be situated just caudal to the middle of the mesonephros while, Leeson and Baxter (1957) in the same animal stated that at $E 15$ the metanephros is present in the pelvis. In our study, we found that the metanephros of rabbit reaches the middle of the mesonephros at E 16 and at E18, the cranial two thirds of the metanephros become cranial to the level of the mesonephros then at E 20 it migrates more cranially to set cranial to the degenerated mesonephros and at E 30, it reaches its permanent position at the middle part of the sublumbar region. The rate of migration is not the same for both kidneys where, from E 12 to $E 14$, both kidneys have the same rate of migration as at E 14, they lie opposite to each other then after, the rate of migration of the right kidney is more rapid than the left one. Con- sequently, at $E 30$ the right kidney is located more cranial, opposite to $2^{\text {nd }}$ $-4^{\text {th }}$ lumbar vertebrae while, the left is situated opposite to $3^{\text {rd }}-5^{\text {th }}$ lumbar vertebrae. These results support the statements of El-Hagri (1967) and Nickel et al. (1973) who stated that, the right kidney of all domestic animals except the pig is somewhat more cranial than the left kidney.

In rabbit embryos, as the right kidney is cranial to the level of the left one, it is located in the renal impression of the liver. This impression is very deep and is formed only by the caudate process of the liver; the right lobe of the liver does not share in its formation. This result is in agreement with Gaber (2013) in rabbit. On the other hand, in the dog, ruminants and horse, the renal impression is formed by the caudate process and the right lobe of the liver (Nickel et al., 1973).

The position of the hilus of the rabbit kidney differs in the different stages of development and it depends upon the degree of rotation of the kidney along its longitudinal axis. At $\mathrm{E}$ 12 , the ureter is connected to the ventral aspect of the primitive metanephros. At E 14, the hilus is located medially as the kidney rotates medially for about 90 degrees along its long axis. At E 18, the hilus is situated somewhat dorsomedially and at E 20, it is placed dorsomedially as a result of its rotation dorsally for about 45 degrees. In the same 
respect, according to Patten (1953) and Arey (1965) the kidneys of human, during their migration, undergo a rotation of 90 degrees, so that the original dorsal border becomes the convex lateral border and the hilus faces medially rather than ventrad. Nickel et al. (1973) stated that, the hilus in horse is located ventrally, that of small ruminants and dog medially but in the left kidney of ox, it is located dorsally. In camel, the hilus is directed ventrally (Imam, 2005).

In rabbit, the renal artery, vein and ureter enter the hilus where, their arrangement to each other differ in different stages of development but this arrangement reaches its adult form at E 23 as, the renal artery is located craniodorsally and the ureter is caudoventrally while the renal vein is inbetween. This arrangement is similar to that present in horse and large ruminants but in small ruminants and dog, the ureter is located in the middle while in pig, the ureter is the most dorsal (El-Hagri, 1967).

According to the present article, the renal pelvis of rabbit is relatively large as the kidney is unipapillary but its lining epithelium differs according to the stage of development from simple columnar to transitional epithelium.

The present results reveal that at $\mathrm{E}$ 15 i.e. the half of gestation period, the terminal branches of the collecting tubules break down the metanephrogenic tissue into small nephrogenic spherules and some of these spherules develop into renal vesicles while in human, the first vesicle is formed at the end of the seventh week (Mishra et al., 2006) and in bovine, nephron induction starts as early as 4-6 weeks gestation (Canfield, 1980). At E 16, some of the renal vesicles become elongated into renal tubules and few of the ends of these tubules, which are close to the terminal branches of the collecting tubules, are continuous with them. The other ends become ampullated and invaginated by mesenchymal mass to form a crescent-shaped glomerular capsule. Differentiation of the proximal and distal convoluted tubules takes place at $E 18$ but in human, the proximal and distal convoluted tubules are clearly first time demarcated at 17 weeks of fertilization (Tank et al., 2012).

This study is in agreement with Aoki (1966) in human who stated that, the early migration of erythrocytes into the vesicular invagination occurs before the glomerular capillaries are completely developed. Similarly, in rabbit we found that, the glomerular capillaries differentiate at E 18 while red blood cells could be observed within the mesenchymal 
mass invaginating the glomerular capsule at E 16 and within the loose mesenchyme surrounding the branches of the ureteric bud and the interlobar mesenchyme earlier at $\mathrm{E}$ 14 and $\mathrm{E} 15$.

At E 23 and $E$ 24, the medullary rays could be detected extending in the cortex towards the renal capsule forming the radiated part of the cortex. Within the medulla, the arteria recti could be observed extending between the collecting tubules towards the renal pelvis. At E 26 and E 28, the interlobar blood vessels could be clearly observed while the renal columns are indistinct consequently; the renal pyramids could not be detected.

According to our observations, positive alkaline phosphatase activity and PAS-positive material were confined to the metanephric corpuscles and the luminal border of the proximal convoluted tubules and the intensity of these reactions increased with increase in fetal age. This similarity in the distribution of alkaline phosphatase and PASpositive material was mentioned before by Moog and Wenger (1952) and Leeson and Baxter (1957) in rabbit. In addition, we found that the collecting tubules showed large masses of intracytoplasmic PASpositive granules throughout all the developmental stages. Localization of alkaline phosphatase in the lu- minal border of the proximal convoluted tubules was also observed by Chida (1993) in rat kidney. Alkaline phosphatase is involved in the reabsorption of glucose in the tubules of the kidney (Lundsgaard, 1935) and the distinctive distribution of the alkaline phosphatase in the luminal border of the proximal convoluted tubules is indicative of functional ability (Bradfield, 1950) that the distinctive distribution of the alkaline phosphatase in the luminal border of the proximal convoluted tubules is indicative of functional ability. Davies (1953) mentioned that, presence of PAS positive granules in the tubule cells of the metanephros may be indicative of protein which had crossed the glomerular membrane and had been reabsorbed by proximal tubule cells. I suggest that reabsorption of protein takes place in the collecting tubules in addition to the proximal convoluted tubules.

\section{References}

Abbas, J.G. and Rabie, F.O. (2012): Histomorphological study of tubular system and collecting tubules in domestic rabbit's fetuses. Kufa journal for veterinary medical sciences, 3 (1): 67-76.

Aoki, A. (1966): Development of the human renal glomerulus. Anat. Rec., 155: 339-352.

Arey, L.B. (1965): Developmental anatomy. A textbook and laboratory 
manual of embryology. $7^{\text {th }}$ Ed. W. B. Saunders Co. Philadelphia. London.

Bradfield, J.R.G. (1950): The localization of enzymes in cells. Biol. Rev., 25: 113-157.

Brewer, N.R. (2006): Biology of the rabbit. Journal of the American Association for Laboratory Animal Science, 45: 8-24.

Canfield, P. (1980): Development of the bovine metanephros. Zbl. Vet. Med. C. Anat. Histol. Embryol., 9: 79-107.

Chida, K. (1993): Immunohistochemical detection of alkaline phosphatase in formalin-fixed and paraffin-embedded rat organs by means of avidin-biotin peroxidase complex method. Okajimas Folia Anat. Jpn., 70 (5): 203-208.

El-Hagri, M.A.A. (1967): Splanchnology of domestic animals. The public organization for books and scientific appliances. Cairo Univ. Press.

Fayez, S.E.; Sayed-Ahmed, A.; Abo-Ghanema, I.I. and Elnasharty, M.A. (2014): Morphogenesis of rabbit kidney pre-and postnatal. Alexandria Journal of Veterinary Sciences, 41: 47-61.

Gaber, W. (2013): Prenatal developmental studies on the rabbit liver. Ph.D. Thesis, Assiut University, Assiut, Egypt.
Gomori, G. (1952): Histochemistery of estrases. Int. Rev. Cytol., 1: 323.

Gruenwald, P. (1943): The normal changes in the position of the embryonic kidney. Anat. Rec., 85: 163176.

Hamilton, J.W. and Mossman, H.W. (1976): Human embryology. 9th Ed. Macmillan Press Ltd., London.

Harris, H.F. (1900): On the rapid conversion of haematoxylin into haematein in staining reactions. J. Appl. Microsc. Lab. Methods, 3: 777-780. Cited by Bancroft et al. (1996).

Huber, G.C. (1905): On the development and shape of uriniferous tubules of certain of the higher mammals. American Journal of Anatomy, 4 (4): 1-98.

Imam, H.M.A. (2005): Early embryonic development of the camel metanephros. Assiut Vet. Med. J., 51: 12-20.

Jackson, C.M. (1909): On the developmental topography of the thoracic and abdominal viscera. Anatomical record, 3 (7): 361-396.

Kaufman, M.H. (1992): The atlas of mouse development. Academic Press. Harcourt Brace Jovanovich, Publishers, London. San Diego. New York. Boston. Sydney. Tokyo. Toronto. 
Leeson, T.S. and Baxter, J.S. (1957): The correlation of structure and function in the mesonephros and metanephros of the rabbit. J. Anat., Lond., 91: 383-390.

Lundsgaard (1935): The effect of phloridzin on the isolated kidney and isolated liver. Skandinav. Arch.

f. Physiol., 72: 265-270.

McGeady, T.A.; Quinn, P.J.; Fitzpatrick, E.S. and Ryan, M.T. (2006): Veterinary embryology. Blackwell Publishing. Ltd, Oxford.

McManus, J.F.A. (1946): Histological demonstration of mucin after periodic acid. Nature (London), 158: 202.

Mishra, S.; Dinesh, A. and Kaul, J.M. (2006): Morphological and morpho-metrical study of human renal development during midgestation period. J. Anat. Soc. India, 55 (2): 5-10.

Moog, F. and Wenger, E.L. (1952): The occurrence of a neutral muco- polysaccharide at sites of high alkaline phosphatase activity. Amer. J. Anat., 90: 339-377.

Nickel, R.; Schummer, A. and Seiferle, E. (1973): The viscera of the domestic mammals. Verlag Paul Parey Berlin. Hamburg.

Patten, B.M. (1953): Human embryology. $2^{\text {nd }}$ Ed., McGraw-Hill Book Company, Inc. New York, Toronto, London.

Rao, B.N. and Padmini, M.P. (2012): Prenatal histogenesis of kindey in human fetuses. International Journal of Basic and Applied Medical Sciences, 2 (2): 144-147.

Sadler, T. W. (2000): Langman's Medical Embryology. $6^{\text {th }}$ Ed., Williams and Wilkins.

Tank, K.C.; Saiyad, S.S.; Pandya, A.M.; Akbari, V.J. and Dangar, K.P. (2012): A study of histogenesis of human fetal kidney. Int J Biol Med Res., 3(1): 1315-1321.

Corresponding author:

e-mail:wafaa.anatomy@gmail.com

Table (1): Age and number of used embryos.

\begin{tabular}{||l|l|l|l|l|l|l|l|l|l|l|l|l|l|l||}
\hline Age (day) & 10 & 11 & 12 & 13 & 14 & 15 & 16 & 18 & 20 & 23 & 24 & 26 & 28 & 30 \\
\hline \hline Number & 4 & 3 & 4 & 3 & 3 & 4 & 2 & 4 & 2 & 1 & 1 & 1 & 1 & 4 \\
\hline
\end{tabular}




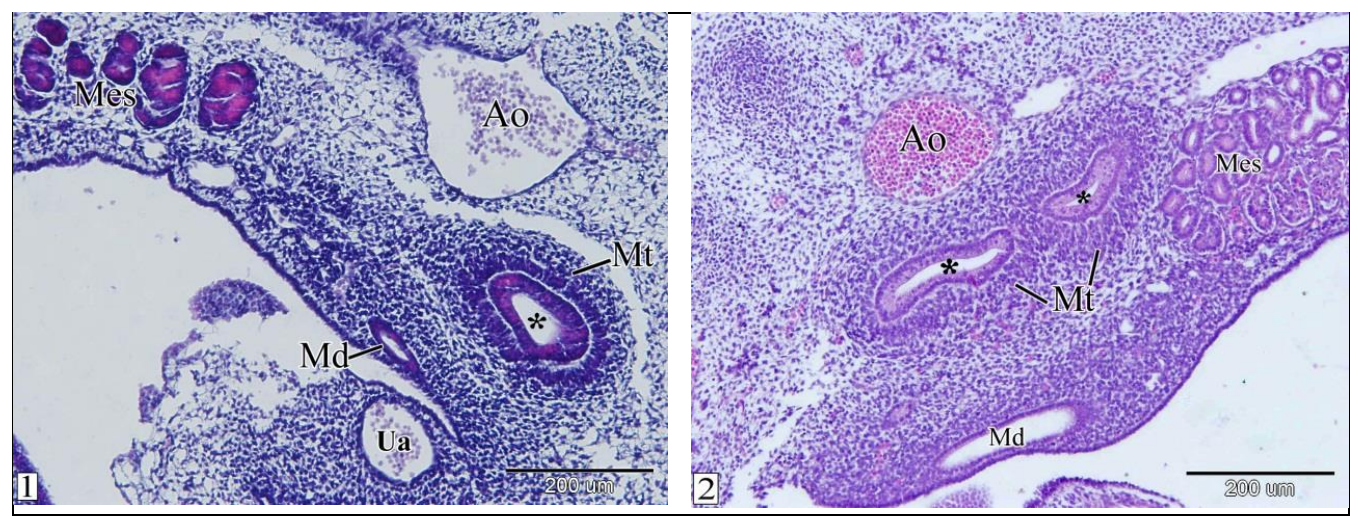

Fig (1): Sagittal section in a rabbit embryo at E 12 showing the primordium of the metanephros. Ureteric bud $\left({ }^{*}\right)$, metanephrogenic tissue (Mt), mesonephric duct (Md), mesonephros (Mes), aorta (Ao) and umbilical artery (Ua). (H\&E, X 100).

Fig (2): Sagittal section in a rabbit embryo at E 13 showing the branches of the ureteric bud $\left({ }^{*}\right)$ surrounded by the metanephrogenic tissue (Mt). Mesonephric duct (Md), mesonephros (Mes) and aorta (Ao). (H\&E, X 100).
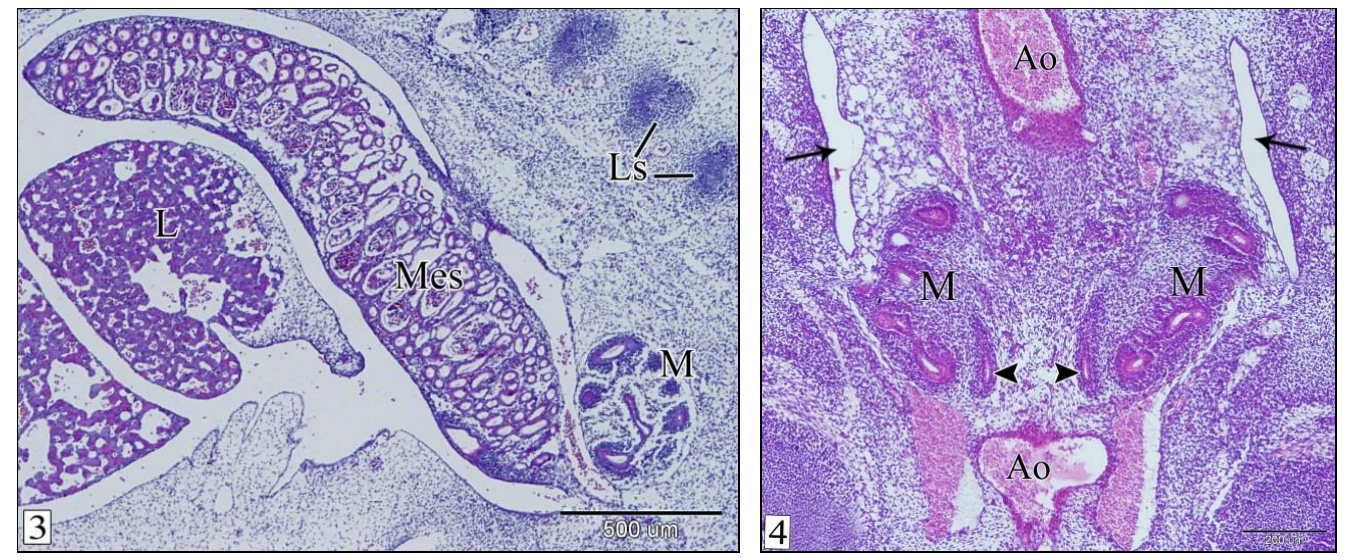

Fig (3): Sagittal section in a rabbit embryo at E 14 showing the metanephros (M) is located dorsal to the caudal end of the mesonephros (Mes). Lumbar somites (Ls) and liver (L). (H\&E, X 40).

Fig (4): Frontal section in a rabbit embryo at E 14 showing the metanephri (M) are bean shaped and located opposite to each other. Ureter (arrowhead), aorta (Ao) and celomic cavity (arrow). (H\&E, X 100). 

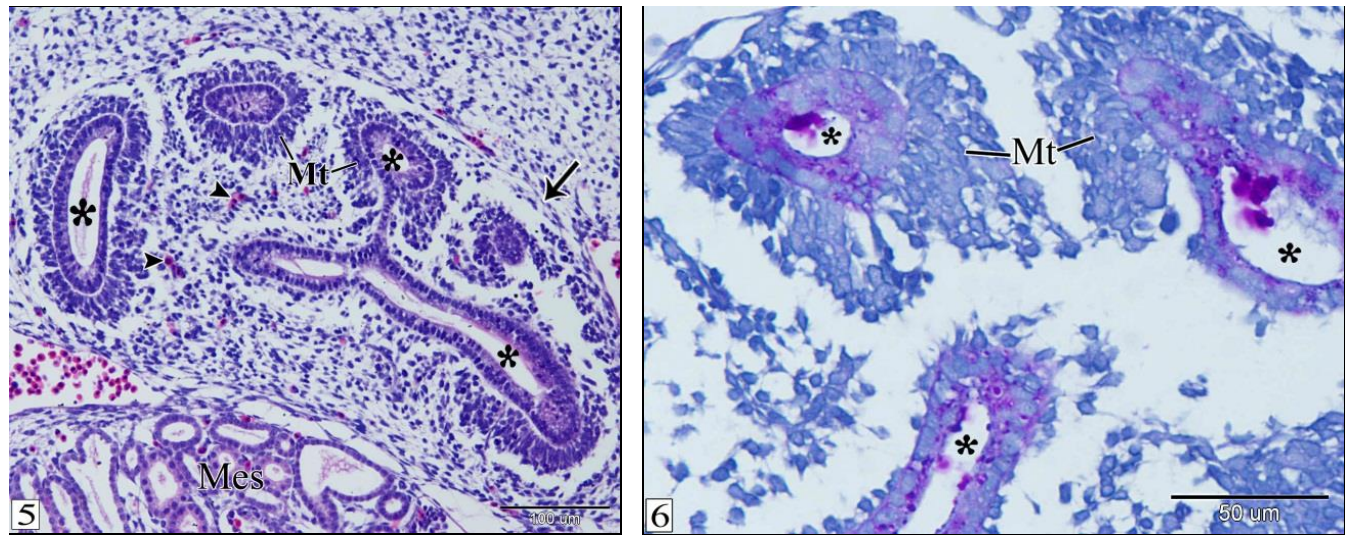

Fig (5): Sagittal section in a rabbit embryo at E 14 showing the structure of the metanephros. Branches of the ureteric bud $\left({ }^{*}\right)$, metanephrogenic tissue (Mt), red blood cells (arrowheads), prospective renal capsule (arrow) and mesonephros (Mes). (H\&E, X 200).

Fig (6): Sagittal section in a rabbit embryo at E 14 showing positive PAS reaction in the branches of the ureteric bud. Notice, the metanephrogenic tissue has negative PAS re-

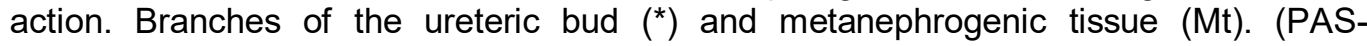
Haematoxylin, X 400).
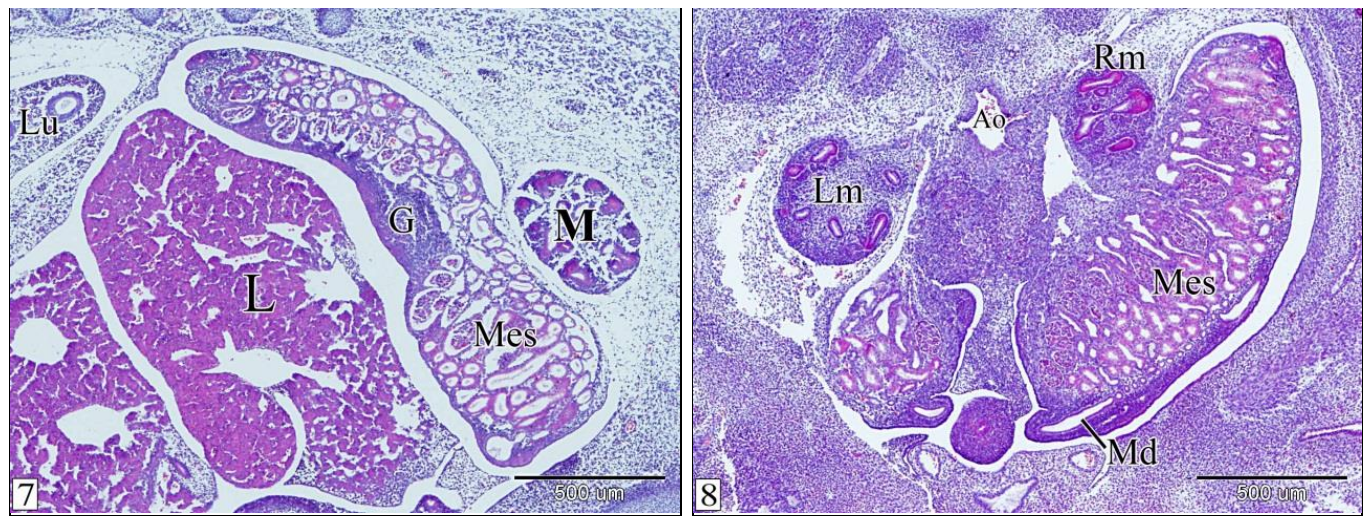

Fig (7): Sagittal section in a rabbit embryo at E 15 showing the metanephros (M) is located dorsal to the mesonephros (Mes), just caudal to its middle. Gonad (G), liver (L) and lung (Lu). (H\&E, X 40).

Fig (8): Frontal section in a rabbit embryo at E 15 showing the right metanephros (Rm) is located somewhat cranial to the left metanephros (Lm). Mesonephros (Mes), mesonephric duct (Md) and aorta (Ao). (H\&E, X 40). 


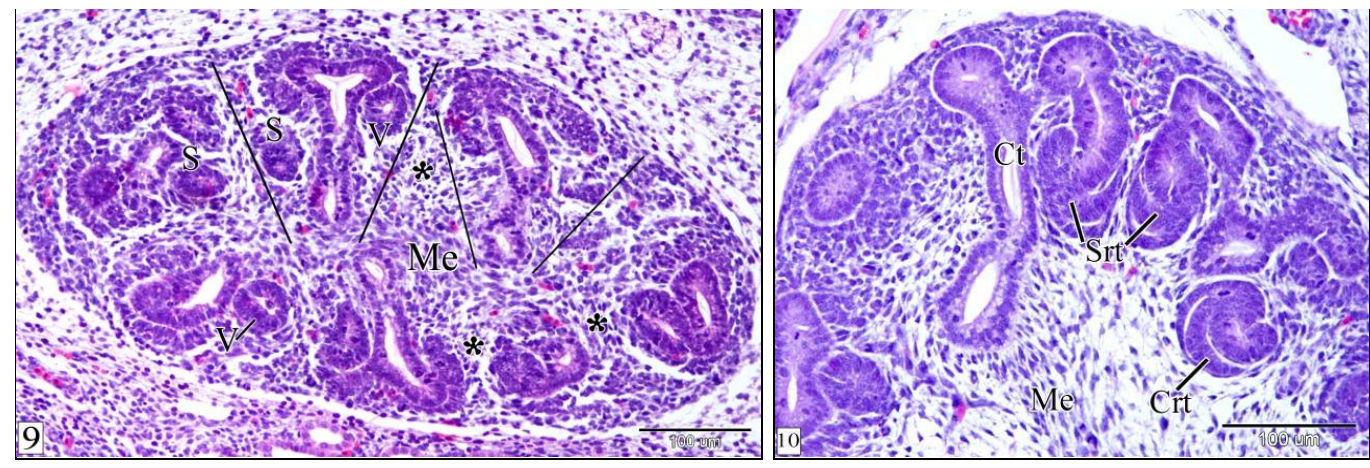

Fig (9): Sagittal section in a rabbit embryo at E 15 showing the structure of the metanephros. Primitive renal lobes (selected areas), interlobar mesenchyme $\left(^{*}\right)$, nephrogenic spherules (S), renal vesicles (V) and medulla (Me). (H\&E, X 200).

Fig (10): Sagittal section in a rabbit embryo at E 16 showing the smooth surface of the metanephros and the different shapes of the renal tubules. Notice, the connection between the S-shape renal tubule (Srt) and the collecting tubule $(\mathrm{Ct})$. Comma-shape renal tubule (Crt) and medulla (Me). (H\&E, X 200).
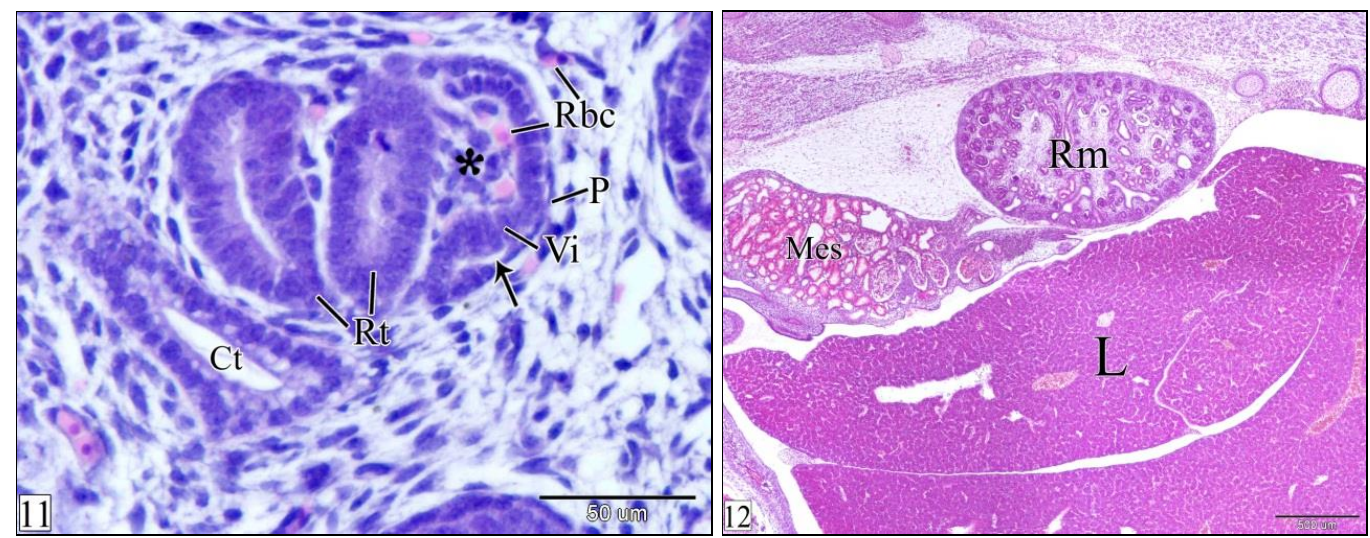

Fig (11): Sagittal section in a rabbit embryo at E 16 showing the ampullated end of the renal tubule invaginated by mesenchymal mass $\left({ }^{*}\right)$ forming glomerular capsule. Parietal layer of glomerular capsule $(\mathrm{P})$, glomerular cavity (arrow), visceral layer of the glomerular capsule $(\mathrm{Vi})$, red blood cells $(\mathrm{Rbc})$, renal tubule $(\mathrm{Rt})$ and collecting tubule $(\mathrm{Ct})$. (H\&E, X 400).

Fig (12): Sagittal section in a rabbit embryo at E 18 showing the right metanephros $(\mathrm{Rm})$ is related ventrally to the liver (L). Mesonephros (Mes). (H\&E, X 40). 

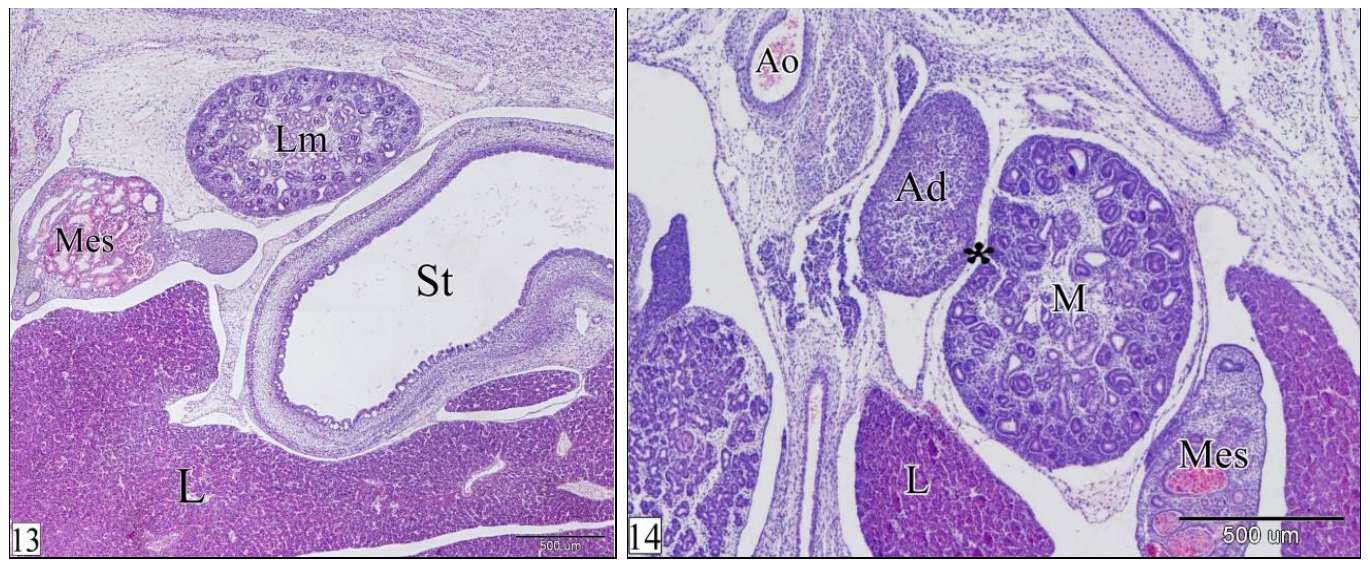

Fig (13): Sagittal section in a rabbit embryo at $E 18$ showing the left metanephros (Lm) is related ventrally to the stomach (St). Mesonephros (Mes) and liver (L). (H\&E, X 40).

Fig (14): Transverse section in a rabbit embryo at E 18 showing the hilus of the metanephros $\left({ }^{*}\right)$ is somewhat dorsomedially located. Metanephros (M), mesonephros (Mes), liver (L), adrenal gland (Ad) and aorta (Ao). (H\&E, X 40).
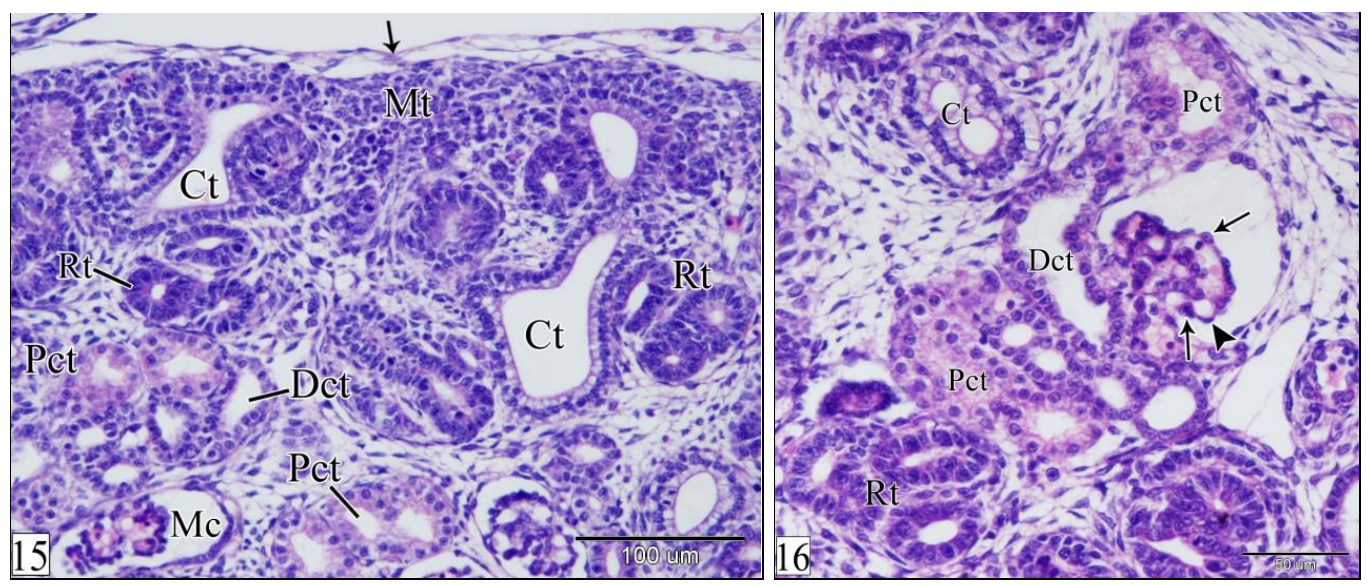

Fig (15): Sagittal section in a rabbit embryo at E 18 showing the structure of the metanephric cortex. Renal capsule (arrow), metanephrogenic tissue (Mt), collecting tubule $(\mathrm{Ct})$, renal tubule (Rt), proximal convoluted tubules (Pct), distal convoluted tubules (Dct) and metanephric corpuscle (Mc). (H\&E, X 200).

Fig (16): Sagittal section in a rabbit embryo at E 18 showing the structure of the deep part of the metanephric cortex. Proximal convoluted tubules (Pct), distal convoluted tubules (Dct), podocytes (arrows), endothelium of the glomerular capillaries (arrow head), renal tubule (Rt) and collecting tubule (Ct). (H\&E, X 400). 

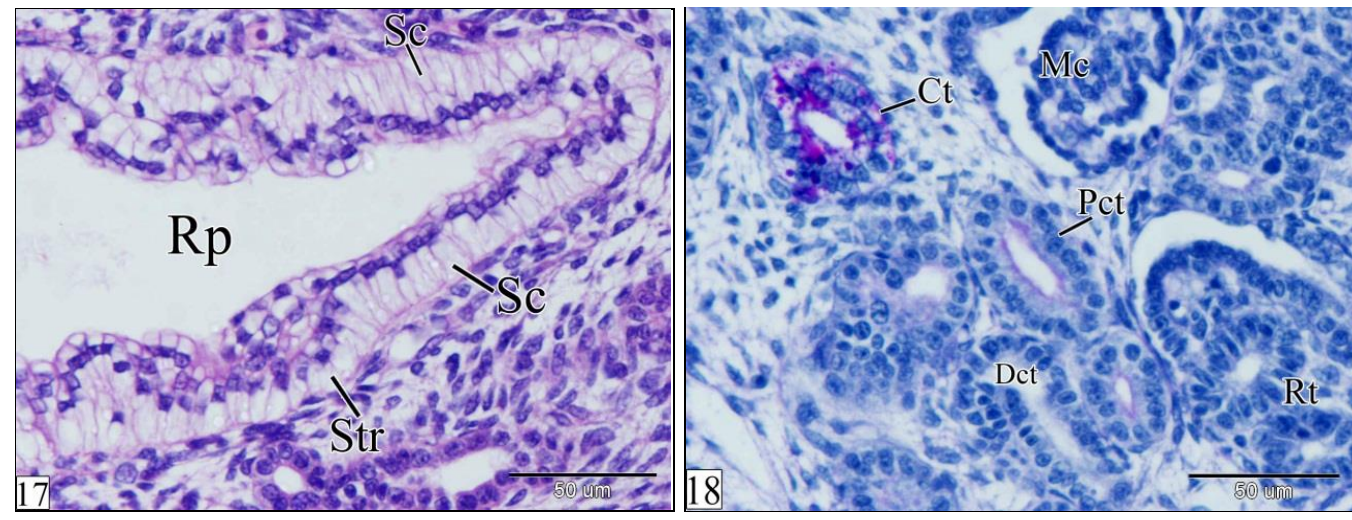

Fig (17): Sagittal section in a rabbit embryo at E 18 showing the lining epithelium of the renal pelvis (Rp). Simple columnar epithelium (Sc) and stratified epithelium (Str). (H\&E, X 400).

Fig (18): Sagittal section in a rabbit embryo at E 18 showing masses of PAS-positive granules in the cytoplasm of the collecting tubules $(\mathrm{Ct})$ and a slight PAS-positive material in the luminal border of some proximal convoluted tubules (Pct). Metanephric corpuscles (Mc), distal convoluted tubules (Dct) and renal tubules (Rt). (PAS-Haematoxylin, $X$ 400).
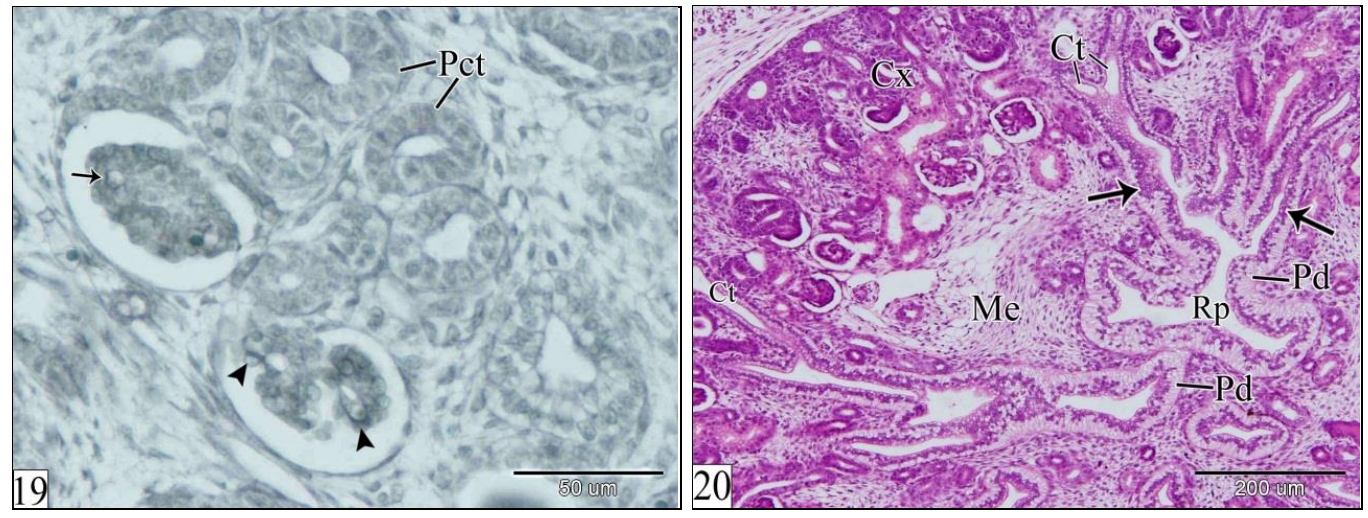

Fig (19): Sagittal section in a rabbit embryo at E 18 showing Slight alkaline phosphatase activity in the luminal border of the proximal convoluted tubules (Pct), the visceral epithelium of the glomerular capsule (arrow heads) and the endothelium of the glomeruli (arrow). (Gomori calcium method, X 400).

Fig (20): Transverse section in a rabbit embryo at E 20 showing the renal pelvis (Rp) gives off papillary ducts (Pd). Stems of the collecting tubules (arrows), collecting tubules (Ct), cortex (Cx) and medulla (Me). (H\&E, X 100). 

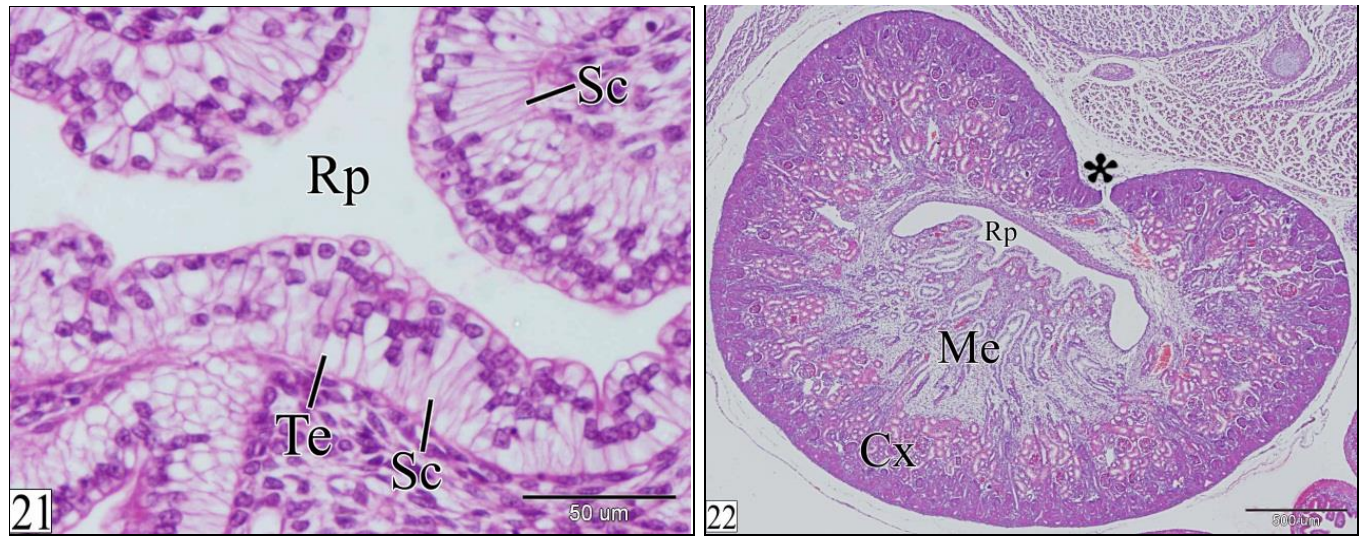

Fig (21): Transverse section in a rabbit embryo at E 20 showing transitional epithelium (Te) in some areas of the lining epithelium of the renal pelvis (Rp). Simple columnar epithelium (Sc). (H\&E, X 400).

Fig (22): Transverse section in a rabbit embryo at E 24 showing increase the demarcation between the cortex (Cx) and the medulla (Me). Renal pelvis (Rp) and hilus $\left(^{*}\right)$. (H\&E, X 40).
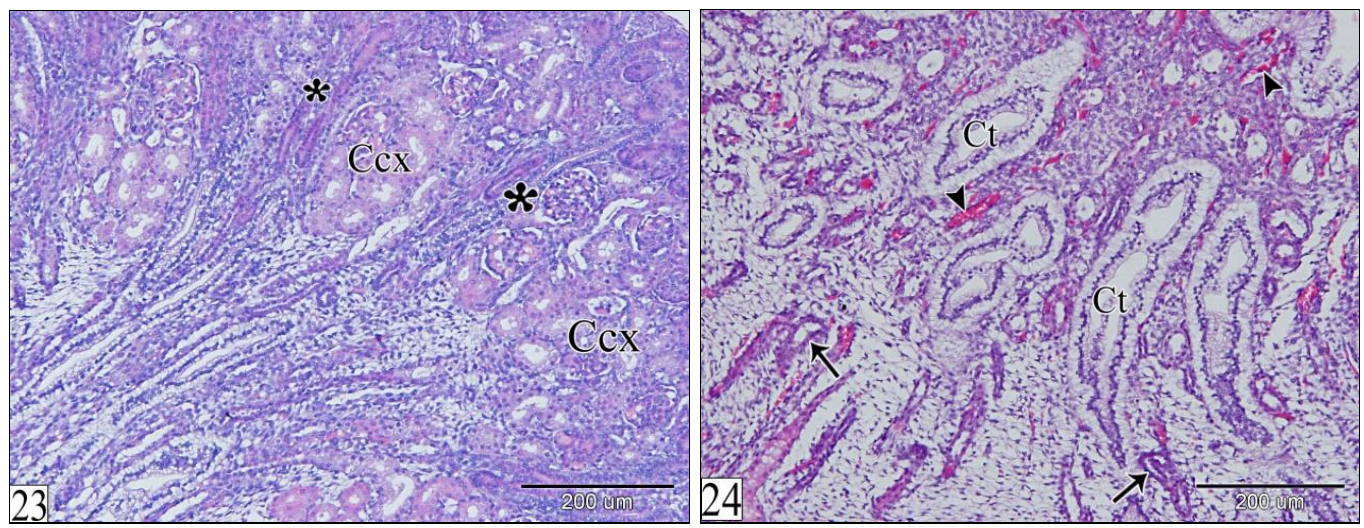

Fig (23): Transverse section in a rabbit embryo at E 24 showing the medullary rays $\left(^{*}\right)$ extending in the cortex. Convoluted part of the cortex (Ccx). (H\&E, X 100).

Fig (24): Transverse section in a rabbit embryo at E 24 showing the arteria recti (arrow heads) between the collecting tubules (Ct). Loop of Henle (arrows). (H\&E, X 100). 

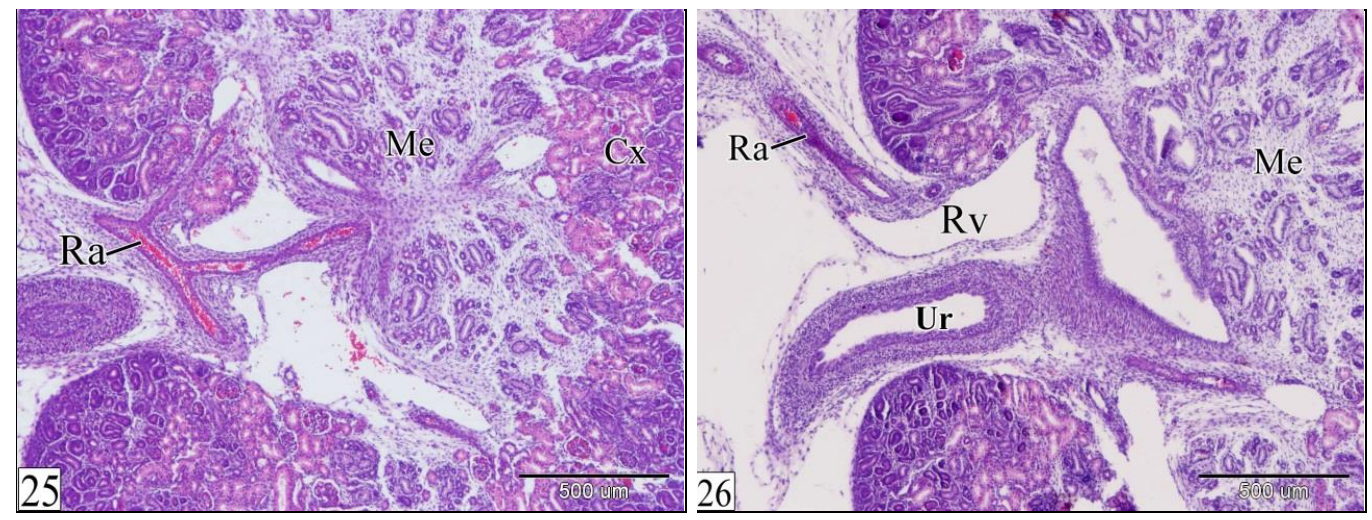

Figs (25 \& 26): Frontal sections in a rabbit embryo at $E 23$ showing the relative position of the main structures in the hilus. Renal artery (Ra), renal vein (Rv), ureter (Ur), cortex (Cx) and medulla (Me). (25) is a more dorsal level than (26). (H\&E, X 40).
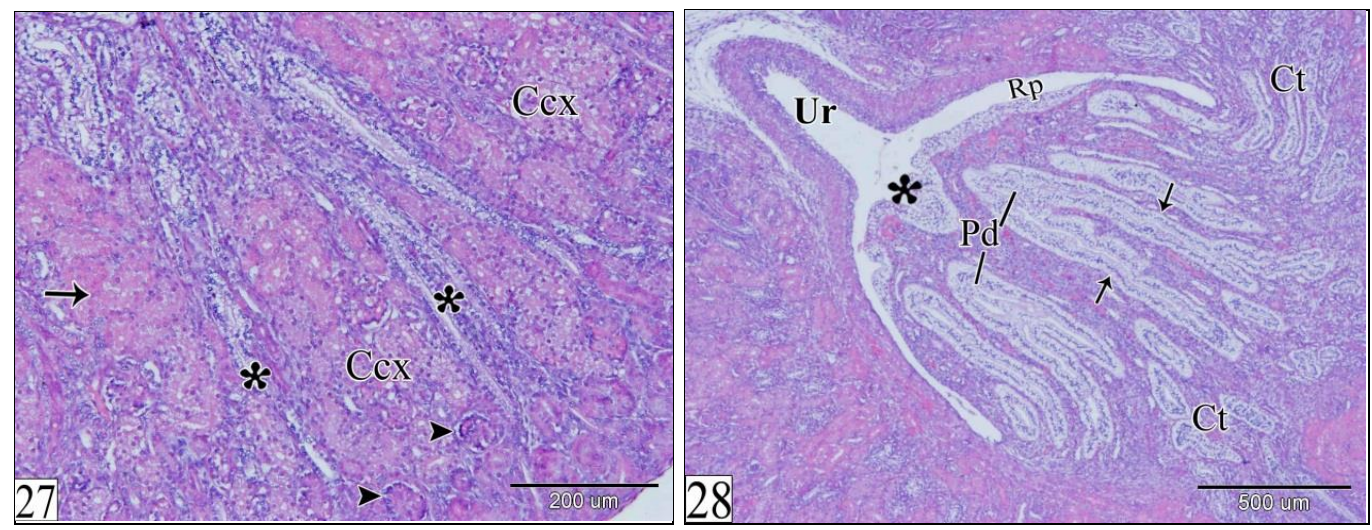

Fig (27): Transverse section in a rabbit embryo at E 26 showing the radiated and convoluted parts of the cortex. Radiated part $\left({ }^{*}\right)$, convoluted part (Ccx), metanephric corpuscles (arrow heads) and convoluted tubules (arrow). (H\&E, X 100).

Fig (28): Transverse section in a rabbit embryo at E 26 showing the meta-nephros is unipapillary. Ureter $(U r)$, renal pelvis $(\mathrm{Rp})$, common renal papilla $\left({ }^{*}\right)$, papillary ducts $(\mathrm{Pd})$, stems of the collecting tubules (arrows) and collecting tubules (Ct). (H\&E, X 40). 

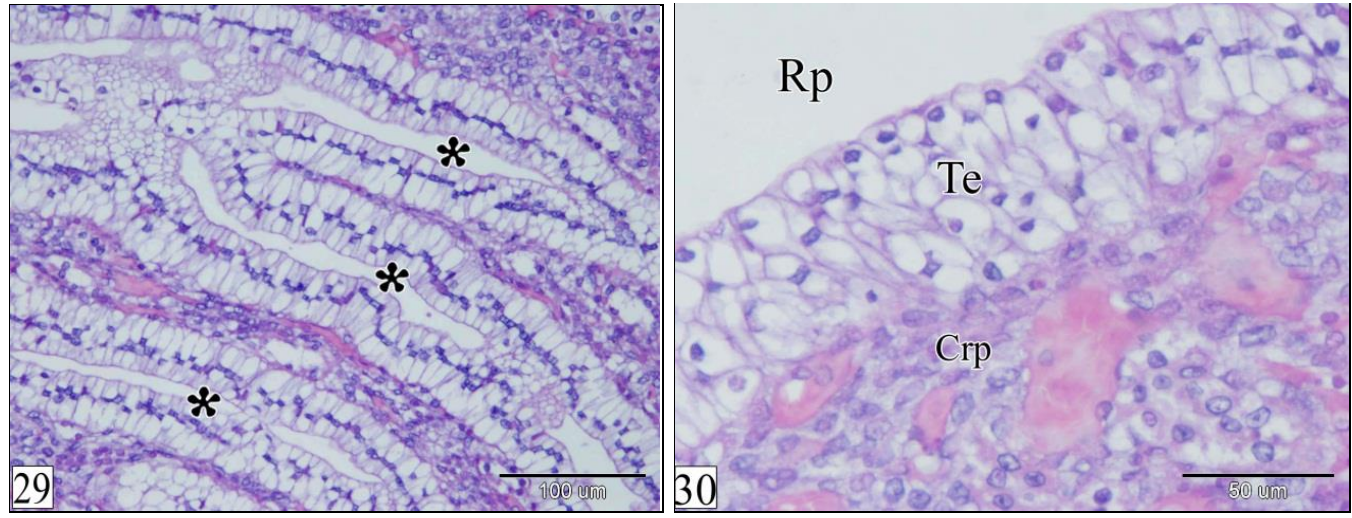

Fig (29): Transverse section in a rabbit embryo at E 26 showing the stems of the collecting tubules $\left({ }^{*}\right)$ are lined by tall columnar epithelium with centrally located nucleus. (H\&E, X 200).

Fig (30): Transverse section in a rabbit embryo at E 26 showing the common renal papilla (Crp) is covered by transitional epithelium (Te). Renal pelvis (Rp). (H\&E, X 400).
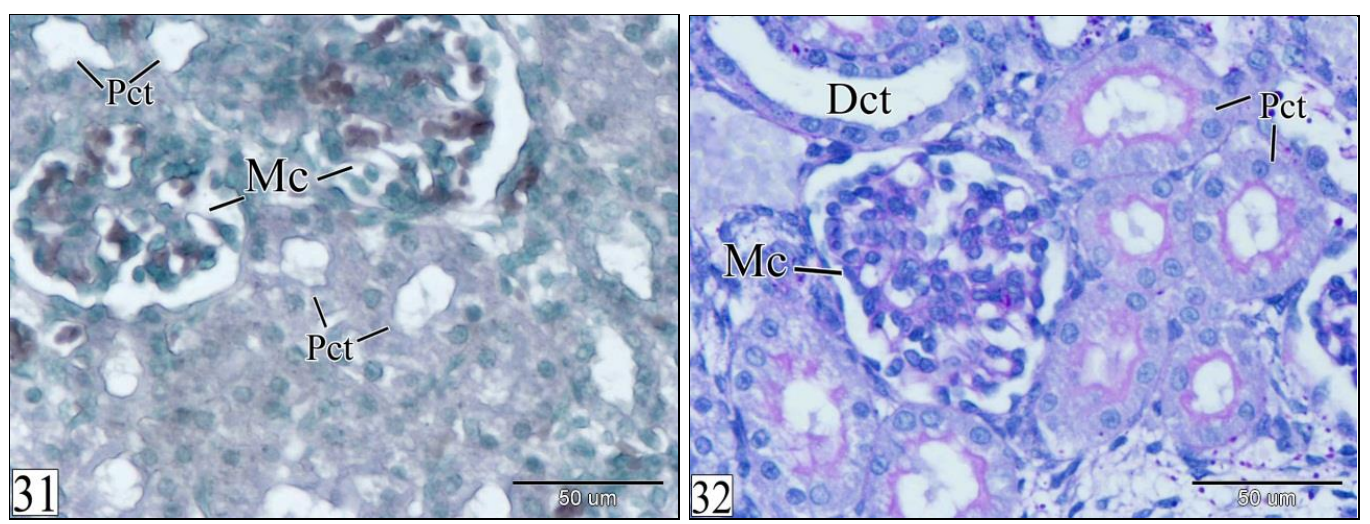

Fig (31): Transverse section in a rabbit embryo at E 24 showing strongly positive alkaline phosphatase activity within the metanephric corpuscles (Mc) and the luminal border of the proximal convoluted tubules (Pct). (Gomori calcium method, X 400).

Fig (32): Transverse section in a rabbit embryo at E 24 showing strong PAS-positive material within the metanephric corpuscles (Mc) and the luminal border of the proximal convoluted tubules (Pct). Notice, the distal convoluted tubules (Dct) are PAS-negative. (PAS-Haematoxylin, X 400). 


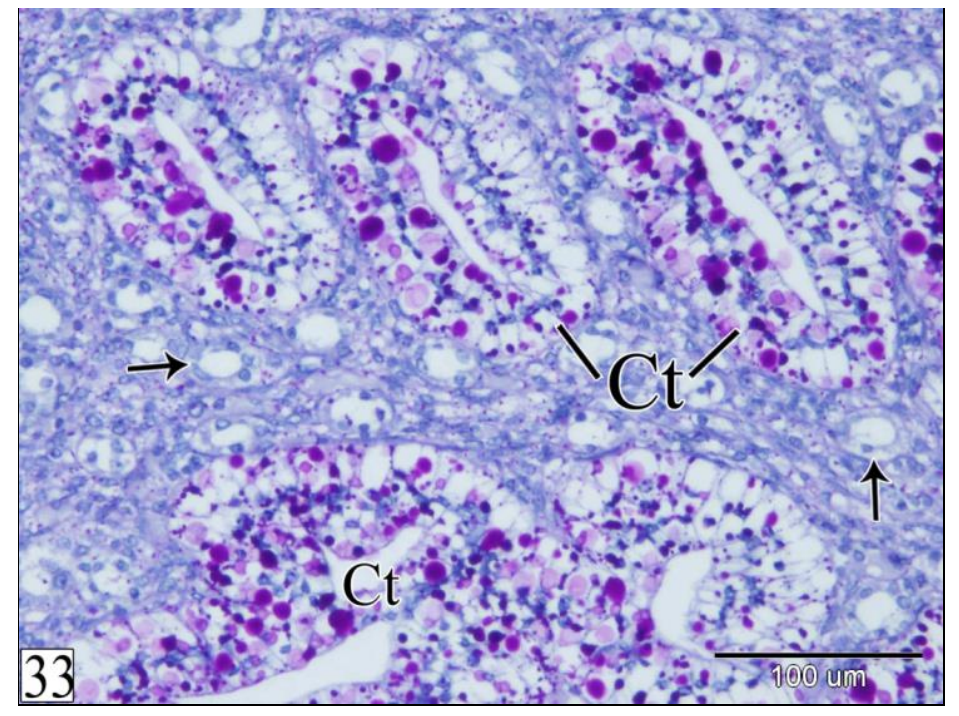

Fig (33): Transverse section in a rabbit embryo at E 26 showing very large masses of PAS-positive granules in the cytoplasm of the collecting tubules (Ct). Notice, the loop of Henle (arrows) is PAS-negative. (PAS-Haematoxylin, X 200).

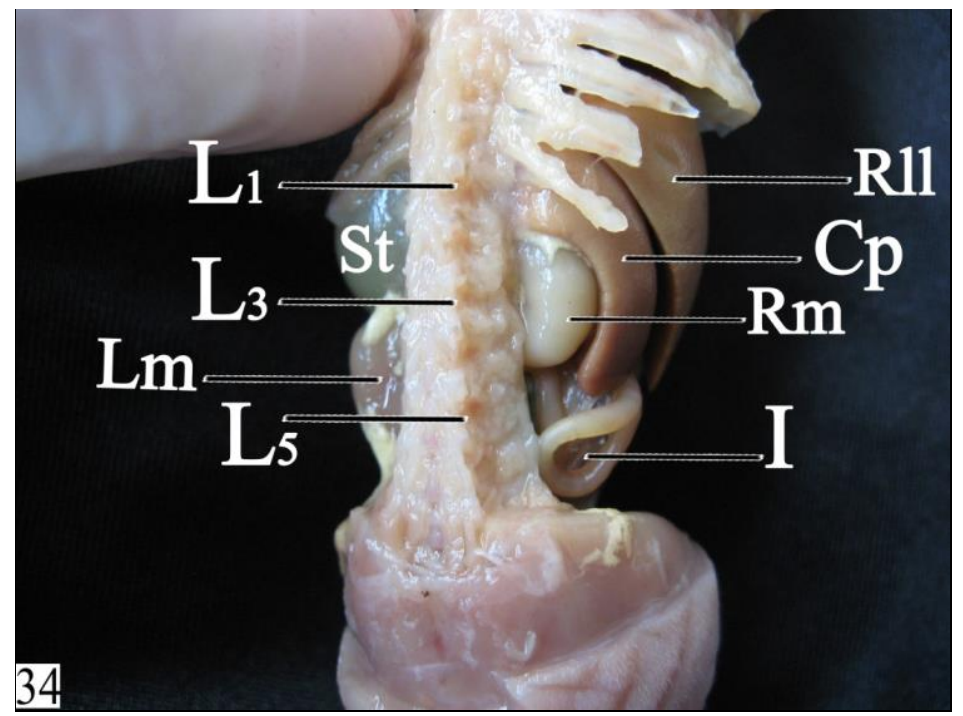

Fig (34): Photograph of a rabbit embryo at E 30 showing the position of the metanephros. Right metanephros $(\mathrm{Rm})$, left metanephros $(\mathrm{Lm})$, right lobe of the liver (RII), caudate process $(C p)$, intestine $(I)$, stomach $(S t)$, first lumbar vertebra $\left(L_{1}\right)$, third lumbar vertebra (L3) and fifth lumbar vertebra (L5). 